\title{
3D Printing of a Scalable Molecular Model and Orbital Kit for Organic Chemistry Teaching and Learning
}

Matthew R. Penny, Zi Jing Cao, Bhaven Patel, Bruno Sil dos Santos, Christopher R. M. Asquith, Blanka R. Szulc, Zenobia X. Rao, Zaid Muwaffak, John P. Malkinson and Stephen

T. Hilton*

UCL School of Pharmacy, 29-39 Brunswick Square, London WC1N 1AX, UK

\section{ABSTRACT}

Three-dimensional (3D) chemical models are a well-established learning tool used to enhance the understanding of chemical structures by converting two-dimensional paper or screen outputs into realistic three-dimensional objects. Whilst commercial atom model kits are readily available, there is a surprising lack of large molecular and orbital models that could be used in large spaces. As part of a program investigating the utility of 3D printing in teaching, a modular size-adjustable molecular model and orbital kit was developed and produced using 3D printing and was used to enhance the teaching of stereochemistry, isomerism, hybridization and orbitals.

\section{ABSTRACT GRAPHIC}

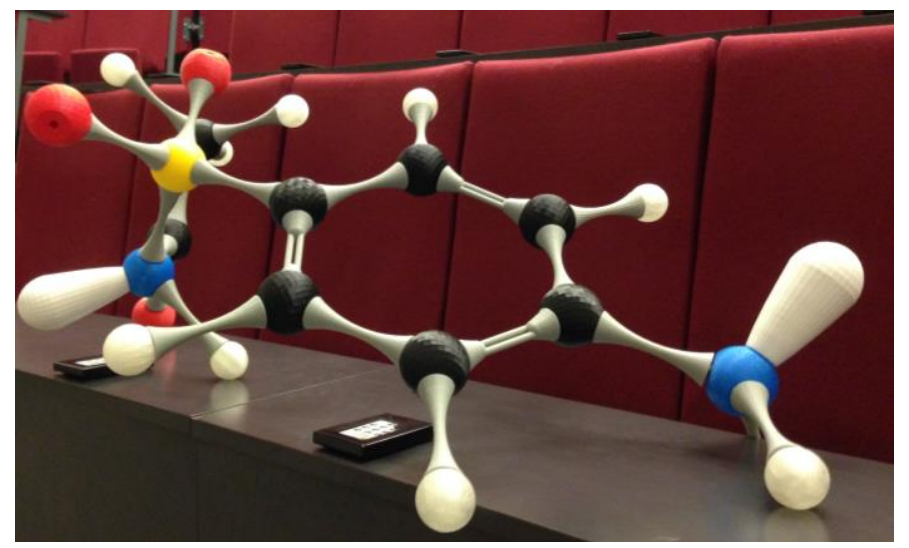

\section{KEYWORDS}

20 Organic Chemistry, Hands-On Learning/Manipulatives, Alkanes/Cycloalkanes, Alkenes, Chirality/Optical Activity, Conformational Analysis, Drugs/Pharmaceuticals, Stereochemistry, Molecular Modeling. 


\section{- INTRODUCTION}

Three-dimensional (3D) models have been an essential kinesthetic pedagogical tool for educators in chemistry due to their ability to facilitate the visualization of molecules in three-dimensions. ${ }^{1-3}$ Due to the difficulty of converting chemical structural representations of molecules from paper into a 3D image in a student's mind, the need for clear physical representations is obvious. As a result, the development of molecular models has received considerable attention for the best part of a century, and commercial models such as Molymod have become widely used by students together with some more esoteric and innovative compositions. ${ }^{4-16}$ The commercial success of model kits, particularly with students of organic chemistry, is due to their modular nature; many molecular models can be assembled, modified and disassembled according to requirements. Unfortunately, due to their small sizes, their usefulness as teaching aids are limited, especially for demonstration purposes during lectures or seminars in large teaching spaces. The construction of bigger models for use with a large audience has been discussed in the literature. However they are often either expensive or lack the flexibility of a modular kit. 17-19 Alongside the development of molecular models there have also been reports describing the construction of molecular orbital models. ${ }^{20-30}$ However, there is a comparative lack of either small- or large-scale orbital models in the commercial area, which is surprising given that students often struggle with the concepts of molecular orbital theory and its application to organic chemistry. ${ }^{31}$

3D printing, or additive manufacturing, has received much attention in the engineering, scientific and lay communities due to its ability to facilitate the rapid development of novel prototypes and is quickly becoming a powerful manufacturing tool. ${ }^{32}$ In recent years this technique has found numerous applications, and synthetic chemists have recently applied 3D printing in both research and education. ${ }^{33-38}$ The ability to construct complex customized objects with ease and at a very low cost $(1 \mathrm{~kg}$ of 
plastic filament currently retails in the region of \$20) has enabled researchers and educators to tailor teaching aids to their lecture courses. This potential has been realized in the area of complex inorganic structures that have been 3D printed as well as models demonstrating abstract concepts in physical and inorganic chemistry. ${ }^{10,39-51}$

As educators in organic chemistry will be all too aware, the concepts that often limit the learning and understanding of students are those that require spatial awareness. Even the most capable students will need to make use of a 3D molecular model in order to reinforce their knowledge and understanding. In this report the application of 3D printing in the development of large customized molecular and orbital models is described. These were used to explain key chemical concepts within the context of a lecture course given to first-year students and complementary 3D printed smaller models were used in follow-up workshop classes. Descriptions of the various models and their relative sizes are described in the supporting information, and pre-prepared 3D printing files are provided free via the author's account on Thingiverse and as supporting information files with this article. ${ }^{52}$

\section{- METHODOLOGY AND APPLICATIONS DESIGN AND CONSTRUCTION OF MODELS}

In order to create a chemistry model set, online published chemical structure designs of models were first investigated. Whilst a 3D model of ethanol on Thingiverse by Rodger proved suitable for one-off small models, it proved unsuitable for larger lecture teaching models due to the less robust round connectors between atoms and bonds. ${ }^{53}$ As such, atom models and bonds were first developed, which it was envisaged could be held together by means of a semi-rigid clip (Figure 1). ${ }^{54}$ Carbon atoms were designed to be large enough (62 $\mathrm{mm}$ diameter) to be visible from the rear of a large lecture theatre for up to 200 people. Bonds to connect atoms were $104 \mathrm{~mm}$ length and were represented as 
shown below for clear visualisation of single, double and triple bonds and rotatable bonds (dimensions and construction shown in Supporting Information). A flat surface for connection of the bonds was created by subtracting a negative image of the clips and a smooth surface from a sphere. Using this approach, atoms with $\mathrm{sp}^{3}, \mathrm{sp}^{2}$ and $\mathrm{sp}$ hybridization (carbon, oxygen, nitrogen), hydrogen atoms, bonds (single, double, triple and rotatable) and clips to join these together were developed and printed with the appropriate colours (Figure 1).
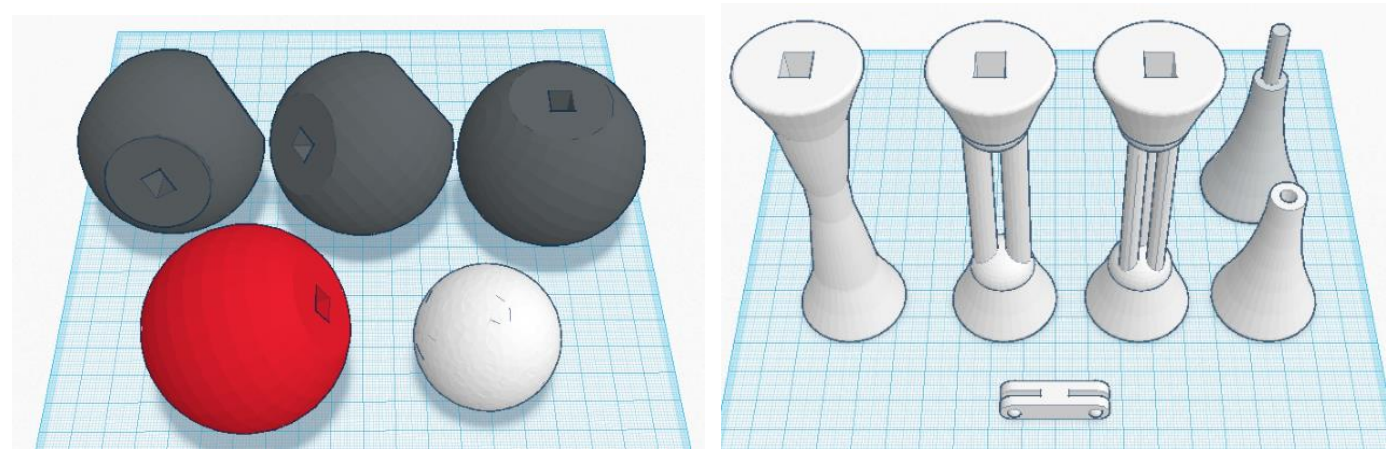

Figure 1. Models of carbon, oxygen, hydrogen, bonds and the clip used to connect these.

Due to the proposed concept of employing the models for use in large teaching spaces, they needed to be both sturdy, and lightweight at the same time. Fortunately, the software accessible to most FDM printers enables a user to select a level of infill (the amount of material placed in the internal structure of the print) that is fit for the required purpose and hence reduces the print time accordingly. As such, all models were printed at a range of infill percentages in polylactic acid (PLA) or acrylonitrile butadiene styrene (ABS) polymers with the exception of the rotatable bonds, which, due to their delicate nature required a higher level of infill at 30\%. The weights, costs, percentage infill and material used for each design are shown in Table 1. 
Table 1. Costs and weights of individual full-size printed modules.

\begin{tabular}{|c|c|c|c|c|c|c|}
\hline Type & Sub-type & $\begin{array}{c}\text { Infill } \\
\%\end{array}$ & $\begin{array}{c}\text { Weight } \\
\text { g }\end{array}$ & Color & $\begin{array}{c}\text { Material } \\
\text { Used }\end{array}$ & $\begin{array}{l}\text { Unit Cost, } \\
\$^{a}\end{array}$ \\
\hline Carbon & $\mathrm{sp}$ & 10 & 20.27 & Black & PLA & 0.53 \\
\hline Carbon & $s p+2 p$ & 10 & 20.27 & Black & PLA & 0.53 \\
\hline Carbon & $\mathrm{sp}^{2}$ & 10 & 20.70 & Black & PLA & 0.55 \\
\hline Carbon & $s p^{2}+2 p$ & 10 & 20.70 & Black & PLA & 0.55 \\
\hline Carbon & $\mathrm{sp}^{3}$ & 10 & 20.92 & Black & PLA & 0.55 \\
\hline Nitrogen & - & 10 & 20.70 & Blue & PLA & 0.55 \\
\hline Nitrogen & $s p^{2}+2 p$ & 10 & 25.49 & Blue & PLA & 0.67 \\
\hline Oxygen & - & 10 & 20.30 & Red & PLA & 0.53 \\
\hline Oxygen & +Lone pairs & 10 & 26.13 & Red & PLA & 0.69 \\
\hline Hydrogen & - & 10 & 9.10 & Clear & PLA & 0.25 \\
\hline Bond & single & 20 & 10.00 & Grey & ABS & 0.26 \\
\hline Bond & double & 20 & 9.90 & Grey & ABS & 0.26 \\
\hline Bond & triple & 20 & 10.20 & Grey & ABS & 0.27 \\
\hline Bond & rotatable & 30 & 10.20 & Grey & ABS & 0.27 \\
\hline Lone pair & - & 10 & 56.83 & White & ABS & 1.49 \\
\hline Lone pair & Pi bond & 10 & 36.94 & White & ABS & 0.97 \\
\hline Lone pair & Pi bond bridge & 10 & 18.65 & White & ABS & 0.49 \\
\hline Lone pair & Pi bond infill & 5 & 8.28 & White & ABS & 0.21 \\
\hline Sigma bond & - & 5 & 34.52 & White & ABS & 0.91 \\
\hline Clip & - & 10 & 1.10 & Green & ABS & 0.03 \\
\hline
\end{tabular}

${ }_{a}$ The price per $\mathrm{kg}$ in USD is 26.33 with the unit cost being a single object of each unit.

During the preparation of atoms, bonds and clips it became evident that different materials were required for different elements as the connectors printed with polylactic acid (PLA) proved too brittle for repeated assembly and disassembly in the models, leading to breakages. Therefore, atoms were printed in either ABS or PLA and bonds and clips in ABS alone.

The development of 3D printed orbital models was more challenging as there were scant reports in the literature about their use and few commercial models available. Their development was therefore explored using 3D printing. $\mathrm{Sp}^{3}$ hybrid orbitals were initially designed that would contain lone pairs of electrons for water and ammonia to gauge the 
correct size for visualisation (Supporting Information). This took three iterations 110 (Supporting Information) to generate the correct size for the large models for use in lectures. These were printed in either ABS or PLA and at $10 \%$ infill to reduce the weight of the final object (Figure 2). For simplicity, a single orbital shape was chosen to be used as a $2 \mathrm{p}$ orbital or as an $\mathrm{sp}^{3}, \mathrm{sp}^{2}$ or $\mathrm{sp}$ hybridized orbital by interchanging with the desired atom of appropriate geometry. The oxygen atom and nitrogen atom were printed using the same $\mathrm{sp}^{3}$ model of the carbon atom, but in a different colour.

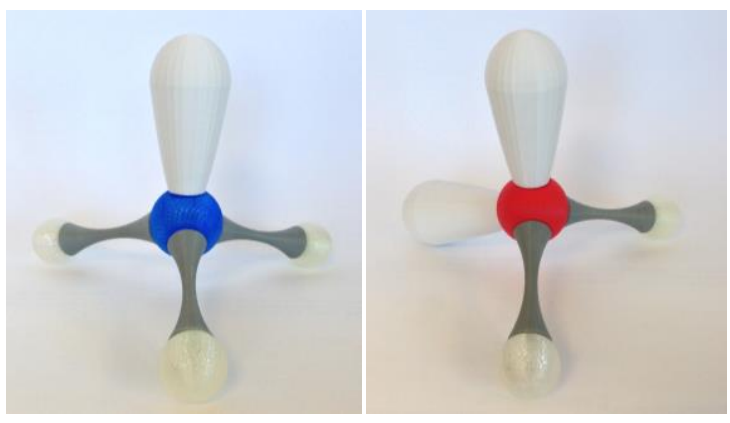

Figure 2. Models of ammonia (left) and water (right) to show the size and scale of the lone pair.

In order to develop carbon atoms with appropriate attachment points for orbitals and pi bonds, a range of atoms was designed and printed (hollow models are shown alongside for clarity of the design with internal clip points) (Figure 3).
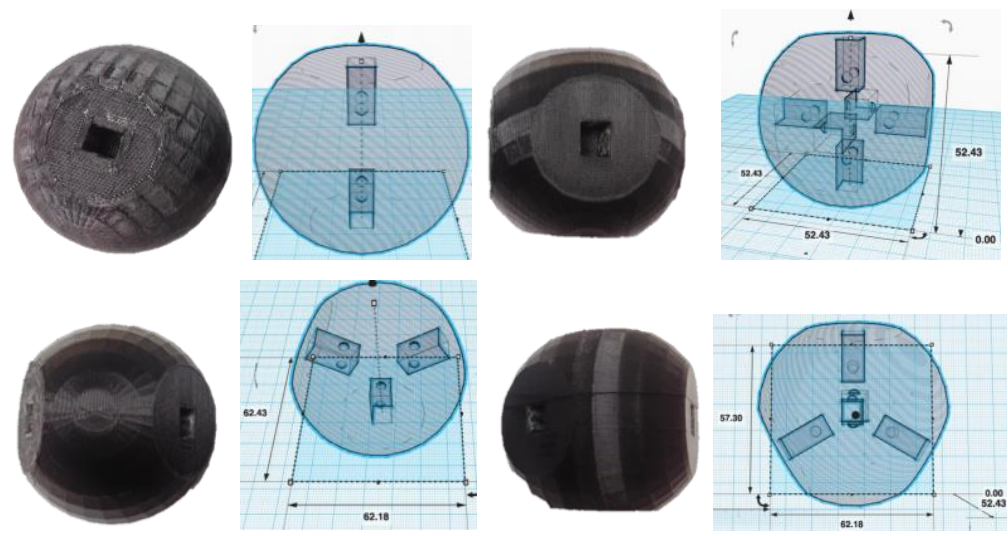

Figure 3. Printed (black) and hollow computer models (grey-striped diagram) of carbon atoms of different hybridizations: $\mathrm{sp}$ (top left), $\mathrm{sp}$ with orbital attachment points (top right), $\mathrm{sp}^{2}$ (lower left) and $\mathrm{sp}^{2}$ with attachment points for orbitals (lower right). 
The second stage of design focused on the correct representation of the bonding orbitals in formaldehyde by firstly constructing a model to show the constituent $\mathrm{p}$ orbitals on the oxygen and carbon atoms. These were linked by a 'bridge' to show side-on orbital overlap leading to formation of a pi bond. The 3D-printed 'bridge' between the p orbitals in the formaldehyde model was designed to demonstrate the basis behind the rigid, nonrotatable carbon-oxygen double bond. These were designed via subtraction of one face of the lone pair and extension of this subtracted section into the pi cloud bridge as shown (further details are provided in the Supporting Information) (Figure 4).

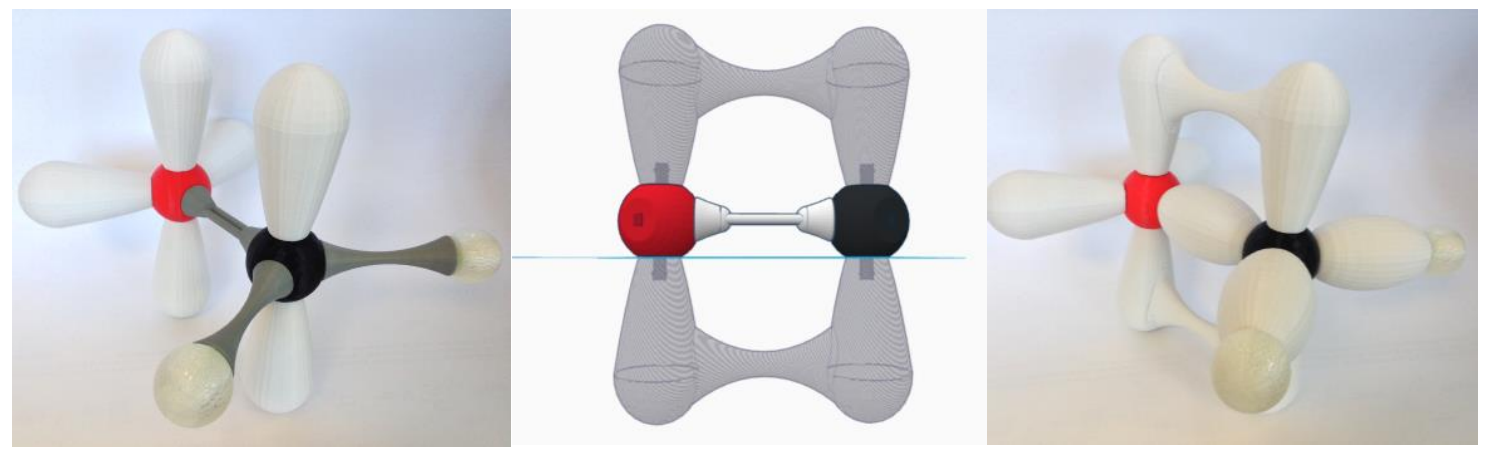

Figure 4. Development of an orbital model of acetone (left). Pi-cloud orbital design model showing the hollow mesh model (centre), and the final model incorporating the pi cloud bridge (right).

Once the models had been printed and evaluated, additional examples were rapidly prepared for workshops where students could benefit from examining and physically interacting with the models. The modular nature of the models allowed a wide range of concepts in organic chemistry to be illustrated as discussed in the following sections.

\section{PRODUCTION OF SMALLER WORKSHOP APPLICABLE MODELS}

Due to the facile method of production using 3D printing, smaller-scale workshopapplicable models (for 50 students working in 10 x groups of 5 led by 4 staff members) could easily be prepared by reducing the size of the models at the point of printing using the software on the 3D printers. Models and corresponding bonds were scaled down in the basic software (MakerBot Desktop) by 50\% to generate the structures shown, with 

$50 \%$ scale, they proved too large to use. However, reducing their size further to $47 \%$ ensured that they were small enough to connect the atoms together (Figure 5 and Supporting Information).

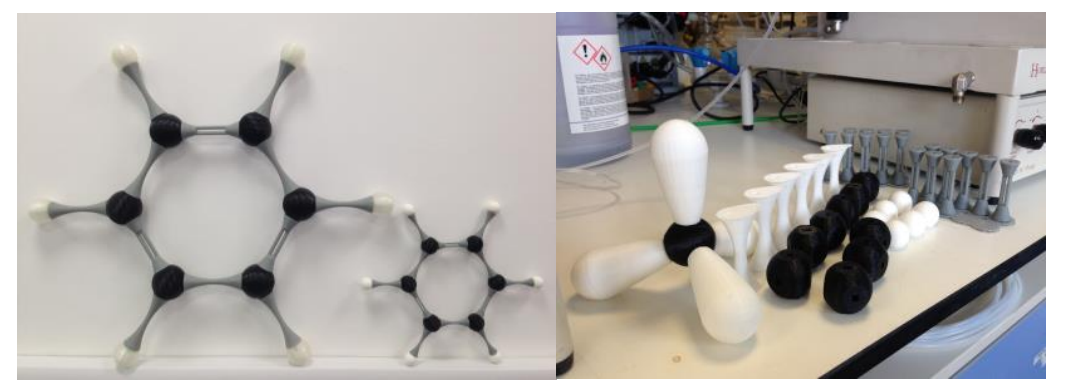

Figure 5. Model of larger-scale benzene against its workshop congener, as well as an illustration of the printing options at $50 \%$ scale.

\section{APPLICATIONS}

\section{Molecular Models}

The initial application focused on the design and production of large, modular 3D molecular models for use in large teaching spaces. These models were designed to explain the concept of molecular shape to a group size of over 180 students as part of a lecture course on structure and bonding (Figure 6). These custom-made models helped to demonstrate fundamental concepts and would have been prohibitively expensive if externally sourced models had been used. The cost of cyclohexane used in this lecture was around $\$ 13$, clearly highlighting the low-cost potential of this approach.

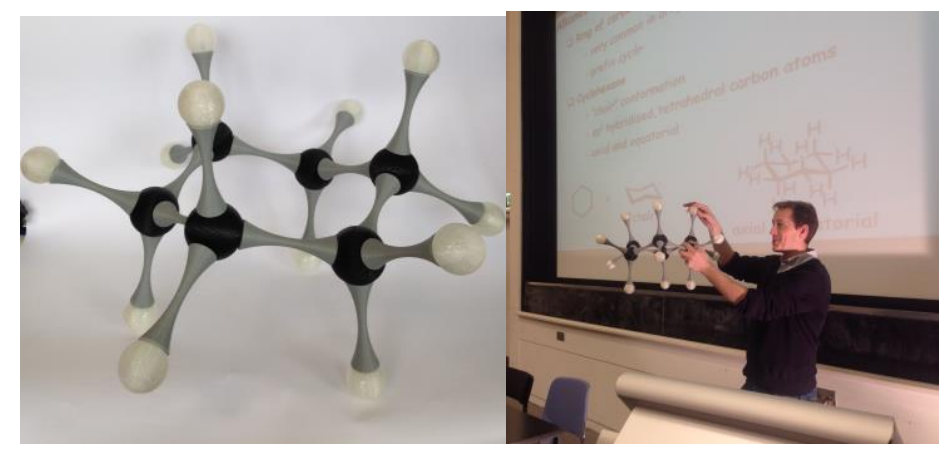

Figure 6. Large-scale models are necessary when presenting to large audiences. In this case a large 3D printed model of cyclohexane (left) is being used to help illustrate its shape and bond angles and to introduce the concept of axial and equatorial orientation of its substituents. Model is fixed in one conformation due to the rigid nature of the clips. 
The study of stereochemistry tests the spatial awareness of chemistry students to a greater extent than any other topic and, as such, has been a focus for the effective use and application of molecular models. ${ }^{55,56}$ This area was therefore an obvious target for this approach to address the fundamentals of geometric isomerism in alkenes and chirality (Figure 7).

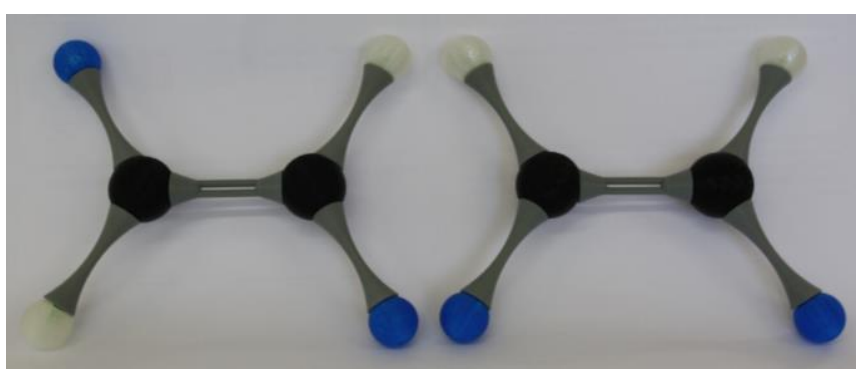

Figure 7. Models of geometric isomers of a simplified alkene showing the trans- and cis-arrangement of atoms that were prepared for demonstration to large groups of students.

There is typically great variability in individual student's innate spatial awareness and this can present difficulties when teaching large groups. Following the discussion of optical and geometric isomerism aided by large 3D printed models during lectures, students were presented with a set of problems in small groups in a workshop setting where mirror image pairs of chiral models were used to aid explanation and the size of each enantiomer can be clearly observed (Figure 8). Students were able to physically manipulate (rotate, align and reorient) the 3D models to help them assign absolute configuration by comparison between molecular structures on paper and 3D models and vice versa. It became evident in the workshop that some students were able to grasp the concept by use of these models and, using peer-supported learning, transferred this information to others. 


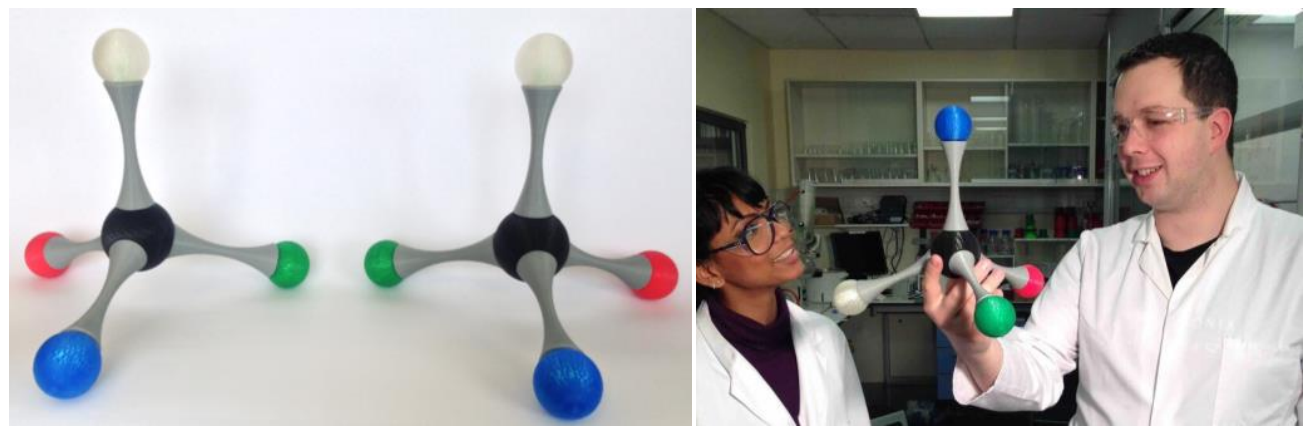

Figure 8. 3D printing has allowed the facile and speedy production of enantiomeric models with which students can interact.

\section{Newman Projections and hybridization}

A common source of confusion for some organic chemistry students is the relationship between conformation and configuration. This topic has great significance in medicinal chemistry and was therefore particularly pertinent to the students' understanding of drug-target interactions. As bespoke models had been created to illustrate configuration, models were created that would allow students to understand bond rotation. ${ }^{34}$ A rotatable bond was designed and developed, so that students could familiarize themselves with Newman projections (Figure 9). As mentioned above, it was decided to print the rotatable bond with a higher infill percentage $(30 \%)$ as it proved to be fairly fragile at lower infill percentages. The eclipsed and staggered forms are shown in Figure 9; by simple manipulation, the interchangeable energy forms can be demonstrated dynamically and are all eminently visible to students. The total cost for this model is less than $\$ 6$, highlighting, again, the low-cost nature of this approach.
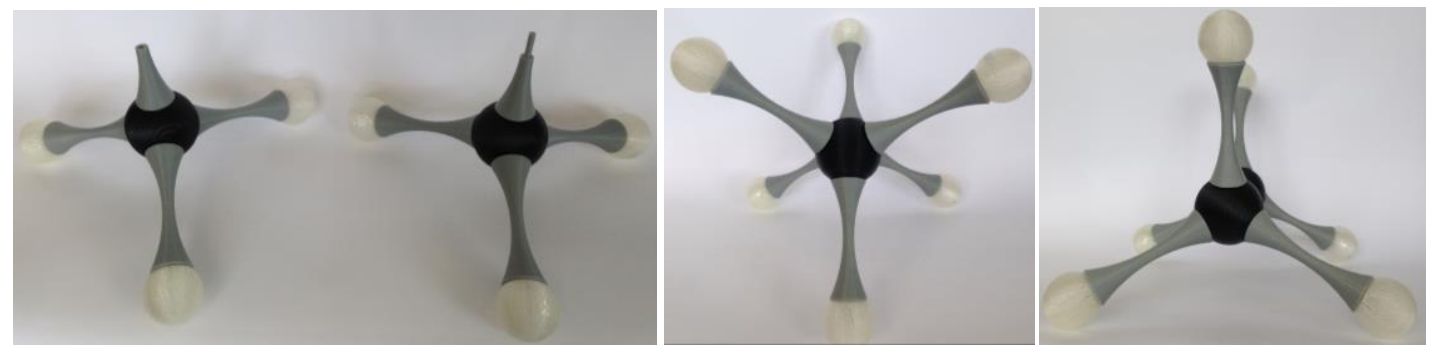

Figure 9. Bespoke rotatable bond to illustrate conformation as exemplified by the staggered and eclipsed forms of ethane. 


\section{Hybridization}

Given the relatively abstract nature of hybridization and its implications to structure and bonding, it was envisaged that the models would have a significant impact on teaching and learning in this area. The relative arrangements of the $\mathrm{sp}^{3}, \mathrm{sp}^{2}, \mathrm{sp}$ and $2 \mathrm{p}$ orbitals in different hybridization states were easily constructed and clearly displayed to students in lectures using a combination of lone pairs and carbon atoms from the orbital kit (Figure 10). The formation of sigma bonds from end-to-end orbital overlap, and pi bonds from side-to-side overlap, were demonstrated in the models of ethene and acetylene (Figure 11).

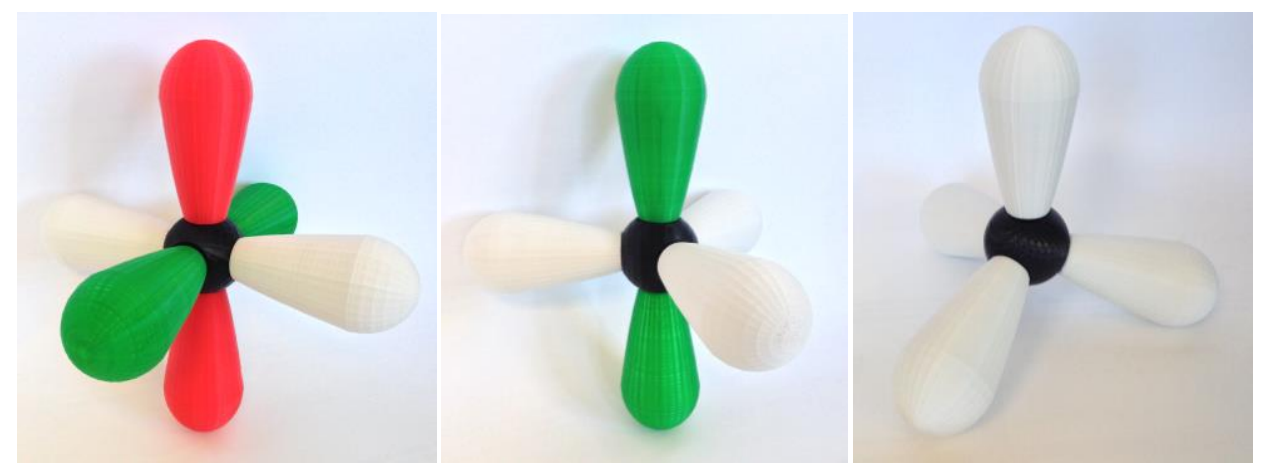

220 Figure 10. $\mathrm{sp}, \mathrm{sp}^{2}$ and $\mathrm{sp}^{3}$ hybridized carbon atoms were designed for use in the teaching of hybridization and to construct molecular orbital models.
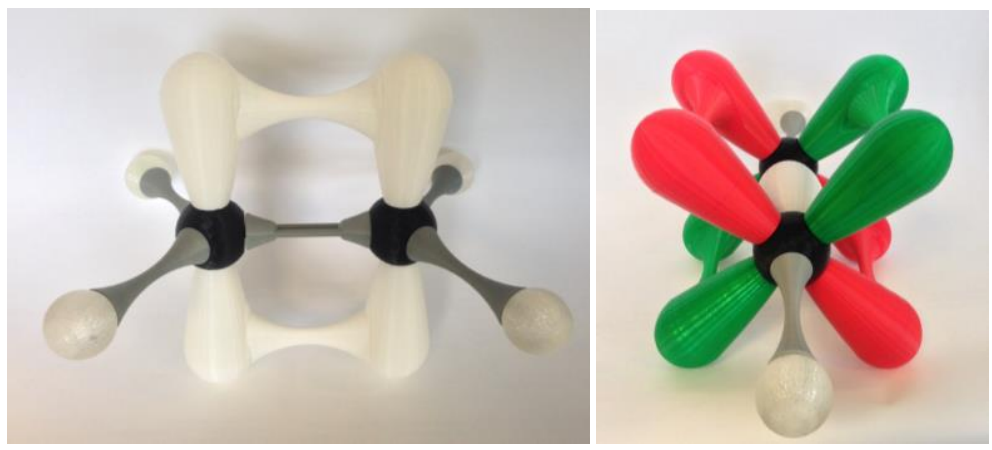

Figure 11. $\mathrm{sp}^{2}$ and $\mathrm{sp}$ hybridized carbon atoms of ethene (left) and acetylene (right) were used to illustrate the orthogonal pi bonds in ethene and acetylene, respectively. 


\section{Aromatic Compounds}

As a result of the fact that the models were initially intended for first-year students, the study of aromatic compounds was of particular importance given their prevalence in medicinal chemistry. As the concepts of hybridization and orbital overlap had been reinforced with the use of large-scale models, the introduction and development of aromaticity was a natural progression for this approach. The large size and ease at which models could be put together and disassembled as required was ideal for displaying aromaticity and the clear extended conjugation to large audiences. NMR spectroscopy and electrophilic aromatic substitution reactions also benefited from this approach where the electron cloud of the aromatic ring could be observed. Furthermore, the model of pyridine provided students with an obvious explanation of its basicity with the clear display of the orthogonal lone pair protruding away from the conjugated pi system (Figure 12).
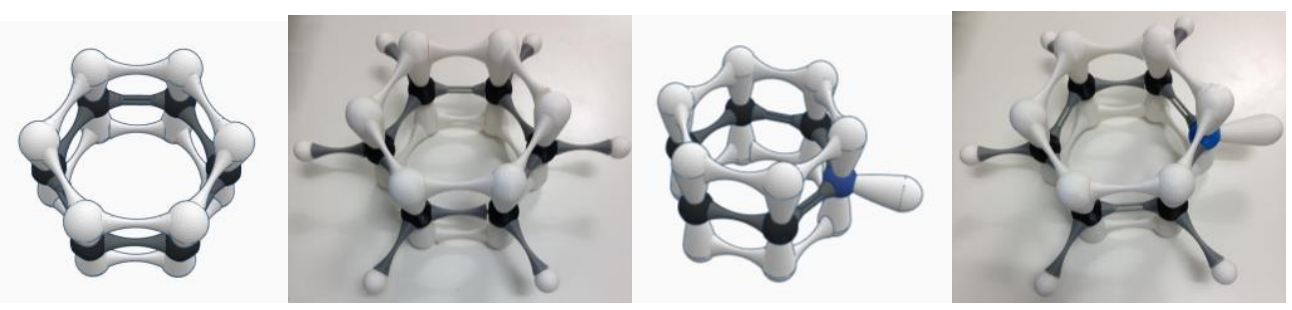

Figure 12. Models of benzene (left) (Tinkercad and printed) and pyridine (right) (Tinkercad and printed) showing the pi cloud of the double bonds and the pi cloud conjugation.

\section{RECEPTION BY STUDENTS}

As part of this study, the impact on the students themselves was of particular interest. Anecdotal feedback from undergraduates was extremely positive as they felt better able to understand structural concepts that they had previously found challenging. The ability for students to physically manipulate models to explore rotation, reorientation and view molecules from alternative perspectives in workshops was well-received. An audience response system was used to collect feedback from more than 180 students at the end of the first term of the module. A simple survey found that $72 \%$ (130) of students agreed $(6 \%$, (11) disagreed) that the models helped with their understanding of structure and bonding 
and $79 \%(149)$ agreed (6\% (11) disagreed) that the use of 3D printed models represented an improvement over 2D images on slides and paper.

\section{CONCLUSIONS}

3D printing has been used to create scalable, modular molecular and orbital models for use as pedagogical aids in teaching organic chemistry with clear positive feedback from students. The infrastructure to support 3D printing within UCL has also been developed, which has allowed the rapid production of models at a very low cost and reproducible models to illustrate fundamental concepts in stereochemistry, configuration and hybridization have been designed. Further studies into the development of more complex structures will be reported in due course and additional models will be uploaded as they are produced. 52

\section{ASSOCIATED CONTENT}

265 Supporting Information

Further detailed pictures and descriptions of the molecules, atoms bonds and orbitals are available via the Internet at http://pubs.acs.org. 3D .stl files of the models described in this publication are available as a complete zip file.

\section{AUTHOR INFORMATION}

Corresponding Author

*E-mail: s.hilton@ucl.ac.uk

\section{ACKNOWLEDGMENTS}

We wish to acknowledge support from UCL Centre for Advanced Learning and

275 Teaching (CALT), UCL Faculty of Life Sciences and an education grant from the School of Life and Medical Sciences at UCL. 


\section{REFERENCES}

1. McCollum, B. M.; Regier, L.; Leong, J.; Simpson, S.; Sterner, S. The Effects of Using TouchScreen Devices on Students' Molecular Visualization and Representational Competence Skills. J. Chem. Educ. 2014, 91 (11), 1810-1817.

2. Copolo, C.; Hounshell, P. B. Using Three-Dimensional Models to Teach Molecular Structures in High School Chemistry. J. Sci. Educ. Technol. 1995, 4 (4), 295-305.

3. Stieff, M.; Ryu, M.; Dixon, B.; Hegarty, M. The Role of Spatial Ability and Strategy Preference for Spatial Problem Solving in Organic Chemistry. J. Chem. Educ. 2012, 89 (7), 854-859.

4. Wade, W. Construction and Use of Models in Chemistry. J Chem. Educ. 1928, 5 (2), 193-196.

5. Minné, N. Molecular Models in Organic Chemistry. J. Chem. Educ. 1929, 6 (11), 1984-1985.

6. Brode, W. R.; Boord, C. E. Molecular Models in The Elementary Organic Laboratory. I. J. Chem. Educ. 1932, 9 (10), 1774-1782.

7. Black, C. E. III; Dole, M. Molecular Models with Free Rotation. J. Chem. Educ. 1941, 18 (9), 424-427.

8. Anker, R. M. Construction of Molecular Models. J. Chem. Educ. 1959, 36 (3), 138-139.

9. Turner, M. Ball and Stick Models for Organic Chemistry. J. Chem. Educ. 1971, 48 (6), 407.

10. Scalfani, V. F.; Vaid, T. P. 3D Printed Molecules and Extended Solid Models for Teaching Symmetry and Point Groups. J. Chem. Educ. 2014, 91 (8), 1174-1180.

11. Gibb Jr, T. R. P.; Bassow, H. Construction of crystal models from styrofoam spheres. J. Chem. Educ. 1957, 34 (2), 99-101.

12. Birk, J. P.; Foster, J. Molecular models for the do-it-yourselfer. J. Chem. Educ. 1989, 66 (12), 1015-1018.

13. Siodlak, D. Building Molecular Models Using Screw-On Bottle Caps. J. Chem. Educ. 2013, 90 $300 \quad$ (9), $1247-1249$.

14. Lam-Leung, W. Y.; Lee, A. W. M. Skeletal Models for Organic Molecules Constructed Easily and Inexpensively. J. Chem. Educ. 1993, 70 (10), 788-789.

15. Niac, G. Balloon Models for Organic Molecules. J. Chem. Educ. 1978, 55 (5), 303.

16. Dragojlovic V. Improving a Lecture-Size Molecular Model Set by Repurposing Used Whiteboard Markers. J. Chem. Educ. 2015, 92 (8), 1412-1414.

17. Lambert, F. L. Molecular models for lecture demonstrations in organic chemistry. J. Chem. Educ. 1953, 30 (10), 503-507.

18. Schultz, H. P. Lecture Demonstration Models of Cycloalkanes. J. Chem. Educ. 1962, 39 (12), 648.

19. Crane, S. C.; Liu, R. S. H. Models for Demonstrating Organic Structures to a Large Audience. J. Chem. Educ. 1986, 63 (6), 516-517.

20. Lambert, F. L. Atomic and Molecular Orbital Models. J. Chem. Educ. 1957, 34 (5), $217-219$.

21. Hoffman K. B. Pi and Sigma Bonding in Organic Compounds. J. Chem. Educ. 1960, 37 (12), 637-638. 
22. Brumlik, G. C. Molecular Models Featuring Molecular Orbitals. J. Chem. Educ. 1961, 38 (10), 502-505.

23. Hoogenboom, R. E. Three-Dimensional Models of Atomic Orbitals. J. Chem. Educ. 1962, 39 (1), 40-41.

24. Martins, G. Atomic Orbital Molecular Models. J. Chem. Educ. 1964, 41 (12), 658-661.

25. Stone, A. H.; Siegelman, I. An Atomic and Molecular Orbital Models Kit. J. Chem. Educ. 1964, $41(7), 395$.

26. Brown, P. Models to Illustrate Orbital Symmetry Effects in Organic Reactions. J. Chem. Educ. 1971, 48 (8), 535-536.

27. Vögtle, F.; Goldschmitt, E. Simple and Inexpensive Orbital Lobe Molecular Models. 1974, 51 (5), 350-351.

28. Stubblefield, C. T. Models to Depict Hybridization of Atomic Orbitals. J. Chem. Educ. 1984, $61(2), 158$.

29. Samoshin, V. V. Orbital Models Made of Plastic Soda Bottles. J. Chem. Educ. 1998, 75 (8), 985.

30. Saieed, A. E. Homemade Models of the Five d-Atomic Orbitals. J. Chem. Educ. 1980, 57 (11), 805-806.

31. Taber, K. S. Compounding Quanta: Probing The Frontiers Of Student Understanding Of Molecular Orbitals. Chem. Educ. Res. Pract. 2002, 3 (2), 159-173.

32. Berman, B. 3-D printing: The new industrial revolution. Bus. Horiz. 2012, 55 (2), $155-162$.

33. Gross, B. C.; Erkal, J. L.; Lockwood, S. Y. Chen, C.; Spence, D. M. Evaluation of 3D Printing and Its Potential Impact on Biotechnology and the Chemical Sciences. Anal. Chem. 2014, 86 (7), 3240-3253.

34. Wijnen, B.; Hunt, E. J.; Anzalone, G, C.; Pearce, J. M. Open-Source Syringe Pump Library. PLoS One, 2014, 9 (9), e107216.

340 35. Halford, B. 3-D Models, Without The Kit. Chem. Eng. News. 2014, 92 (20), 32-33.

36. Stefanov, B. I.; Lebrun, D.; Mattsson, A.; Granqvist, C. G.; Österlund, L. Demonstrating Online Monitoring of Air Pollutant Photodegradation in a 3D Printed Gas-Phase Photocatalysis Reactor. J. Chem. Educ. 2015, 92 (4), 678-682.

37. Dragone, V.; Sans, V.; Rosnes, M. H.; Kitson, P. J.; Cronin, L. 3D-printed devices for continuous-flow organic chemistry. Beilstein J. Org. Chem. 2013, 9, 951-959.

38. Tyson, A. L.; Hilton, S. T.; Andreae, L. Rapid, simple and inexpensive production of custom 3D printed equipment for large-volume fluorescence microscopy. Int. J. Pharm. 2015, 494, 651656.

39. Kitson, P. J.; Macdonell, A.; Tsuda, S.; Zang, HY., Long, D-L.; Cronin, L. Bringing Crystal Structures to Reality by Three-Dimensional Printing. Cryst. Growth Des. 2014, 14 (6), 27202724. 
40. Chen, T-H.; Lee, S.; Flood, A. H.; Miljanić, O. Š. How to print a crystal structure model in 3D. CrystEngComm. 2014, 16 (25), 5488-5493.

41. Casas, L.; Estop, E. Virtual and Printed 3D Models for Teaching Crystal Symmetry and Point Groups. J. Chem. Educ. 2015, 92 (8), 1338-1343.

42. Blauch, D. N.; Carroll, F. A. 3D Printers Can Provide an Added Dimension for Teaching Structure-Energy Relationships. J. Chem. Educ. 2014, 91 (8), 1254-1256.

43. Lolur, P.; Dawes, R. 3D Printing of Molecular Potential Energy Surface Models. J. Chem. Educ. 2014, 91 (8), 1181-1184.

44. Teplukhin, A.; Babikov, D. Visualisation of Potential Energy Function Using an Isoenergy Approach and 3D Prototyping. J. Chem. Educ. 2014, 92 (2), 305-309.

45. Robertson, M. J.; Jorgensen W. L. Illustrating Concepts in Physical Organic Chemistry with 3D Printed Orbitals. J. Chem. Educ. 2015, 92 (12), 2113-2116.

46. Wedler, H. B.; Cohen, S. R.; Davis, R. L.; Harrison, J. G.; Siebert, M. R.; Willenbring, D.; Hamann, C. S.; Shaw, J. T.; Tantillo, D. J. Applied Computational Chemistry for the Blind and Visually Impaired. J. Chem. Educ. 2012, 89 (11), 1400-1404.

47. Scalfani, V. F.; Turner, C. H.; Rupar, P. A.; Jenkins, A. H.; Bara, J. E. 3D Printed Block Copolymer Nanostructures, J. Chem. Educ. 2015, 92 (11), 1866-1870.

48. Stone-Sundberg, J.; Kaminsky, W.; Snyder, T.; Moeck, P. 3D Printed Models of Small and Large Molecules, Structures and Morphologies of Crystals, as well as their Anisotropic Physical Properties. Cryst. Res. Technol. 2015, 50, 432-441.

49. Lickiss, M. Pictures of the Molecular World, Educ. Chem. 2015, 18. ISSN 0013-1350.

50. Flint, E. B. Teaching Point-Group Symmetry with Three-Dimensional Models. J. Chem. Educ. 2011, 88 (7), 907-909.

51. Rossi, S.; Benaglia, M.; Brenna, D.; Porta, R.; Orlandi, M. Three Dimensional (3D) Printing: A Straightforward User-Friendly Protocol to Convert Virtual Chemical Models to Real-Life Objects. J. Chem. Educ. 2015, 92 (8), 1398-1401.

52. Molecular Models collection - Thingiverse; http://www.thingiverse.com/st_hilton/designs (Accessed June 27, 2017).

53. Chemistry Molecule construction set by andrewar - Thingiverse; http://www.thingiverse.com/thing:21398 (Accessed June 27, 2017).

54. Eleventh Doctor Who's Sonic Screwdriver Snap Together by MakerBlock - Thingiverse; http://www.thingiverse.com/thing:68464 (Accessed June 27, 2017).

55. Feldman, M. R. Models for Illustrating Chirality at Two Centers. J. Chem. Educ. 1984, 61 (12), 1050.

56. Nave, P. M. Natural and Unnatural Models for Illustrating Chirality at Two Centers. J. Chem Educ. 1991, 68 (12), 1028-1029. 